\title{
Un outil informatique d'aide à la gestion intégrée de la ressource en eau: le logiciel AGIRE Exemples d'application*
}

\author{
J. Rietjens, J. Gailhard, Ph. Gosse, K. Malatre, \\ T. Royer, C. Sabaton, F. Travade(1) \\ (1) Électricité de France, Direction des Études et Recherches, \\ Département Environnement, 6 quai Watier, 78400 Chatou.
}

Résumé. - Alors qu'avec la nouvelle loi sur l'eau du 3 janvier 1992, le cadre législatif oriente les différents acteurs de l'eau vers des procédures de concertation, on constate le manque d'outils opérationnels pour la mise en place localement de telles démarches. Ce n'est pourtant pas une tâche aisée dans la mesure où les relations entre les usagers sont souvent conflictuelles, notamment en situation de pénurie. La sensibilisation de ces acteurs aux besoins des uns et des autres, étape indispensable pour mener à bien le dialogue, requiert donc un effort en matière de communication.

Dans ce contexte, la Direction des Etudes et Recherches (DER) d'EDF développe depuis 1991 un outil informatique d'aide à la gestion intégrée de la ressource en eau : le logiciel AGIRE. La première version de ce logiciel (version 1.0) est aujourd'hui achevée : il s'agit d'une version monodimensionnelle, en mode permanent et à pas mensuel.

Cet outil se présente sous la forme d'un logiciel interactif, facilement utilisable pour un non initié, et qui permet au gestionnaire de visualiser les conséquences, à la fois sur le milieu aquatique el sur la satisfaction des usagers, de différents scénarios d'aménagement et de gestion du bassin. Ces scénarios peuvent porter sur le niveau de développement ou d'équipement des usages agricoles, industriels ou domestiques, sur les schémas de circulation d'eau (lâchers de barrages amont, transfert d'eau entre rivières,..), sur les contraintes environnementales (débit de salubrité par exemple), sur les aléas climatiques...

Cet outil informatique, qui fonctionne sur station de travail, est bâti autour :

- d'un outil de simulation reposant sur une bibliothèque de modèles numériques;

- d'un outil de visualisation développé à partir du système d'information géographique ARC INFO.

Une première application sur deux rivières du Sud-Ouest de la France (Gave de Pau et Echez), réalisée dans le cadre d'une convention entre EDF-DER, l'Agence de l'Eau Adour-Garonne, le Ministère de l'Environnement et l'Institution Interdépartementale pour l'Aménagement Hydraulique du Bassin de l'Adour, est opérationnelle à l'Agence de l'Eau Adour-Garonne.

- Article présenté au Colloque d'Hydroécologie des 31 mai-1or juin 1994 - Paris - France. 
Quelques exemples de résultats de scénarios (augmentation des surfaces irriguées, amélioration du rendement des stations d'épuration existantes, hypothèse d'un transfert d'eau entre rivières en période estivale) permettent d'illustrer l'utilisation possible de la version 1.0 du logiciel AGIRE.

Mots-clés. - Gestion de l'eau, modélisation, SIG, Gave de Pau, Echez.

Abstract. - While legislation under the new water law of january 3, 1992, recommends regular consultation among the various actors, there is an obvious need for operational tools to help in locally setting up the procedures. This is not an easy task, however, given that relations among water users are often conflictive, particulary in cases of shortage. Making all sides aware of the needs of the others, which is indispensable to constructive dialogue, therefore requires an effort to improve communication.

It is in this context that, since 1991, the EDF Research and Development Division (DER) has been developing software to aid in integrated water resource management: AGIRE. The first version (version 1.0) is now complete. It is one-dimensional version that functions in permanent mode on a one-month time step.

AGIRE is interactive software, easily accessible to the non-expert, which enables those interested in water management to visualize the consequences both on the aquatic environment and on user satisfaction of various scenarios regarding installation and management of a river basin. These scenarios may bear on the level of development or equipment for agricultural, industrial or domestic purposes; patterns of water circulation (releases from upstream dams, transfer from one river to another, etc.); environmental constraints (minimum acceptable discharge for sanitary reasons, for example); climatic events, etc.

The software, run from a workstation, is built around:

- a simulation tool based on a library of numeric models;

- a visualization tool developed with aid of the ARC INFO geographical information system.

Its first application to two rivers in southwest France (Gave de Pau and Echez) in the framework of an agreement between EDF-DER, the Adour-Garonne River Agency, the Ministry of the Environment and the Interdepartemental Commission on hydraulic development of the Adour Basin, is now operational at the Adour-Garonne River Agency.

This paper presents some sample scenarios (increase in irrigated areas, improved efficency of existing water treatment stations, hypothesis of water transfer between rivers in summer) which illustrate the possible use of version 1.0 of AGIRE.

Key words. - Water Ressource Management, simulation tool, GIS, Gave de Pau, Echez.

\section{INTRODUCTION}

Alors qu'avec la nouvelle loi sur l'eau du 3 janvier 1992, le cadre législatif oriente les différents acteurs de l'eau vers des procédures de concertation, on constate le manque d'outils opérationnels pour la mise en place lo- calement de telles démarches. Ce n'est pas une tâche aisée dans la mesure où les relations entre les usagers sont souvent conflictuelles, notamment en situation de pénurie. Cette démarche implique le choix de "priorités", choix qui passe par :

- la définition d'objectifs de qualité pour le milieu aquatique 
- un inventaire des besoins des différents usagers de l'eau

- une concertation entre les acteurs locaux et la définition d'une règle de partage de la ressource.

Pour un tel objectif, le recours aux outils informatiques, associés à des bases de données suffisament riches, devient une aide précieuse, d'autant que les technologies disponibles aujourd'hui, tant dans les domaines des outils de simulation ou des Systèmes d'Information Géographique (SIG), permettent de concevoir des systèmes informatiques adaptés à la problématique de la gestion intégrée d'un bassin versant (Fourcade et al., 1993).

Dans ce contexte, la Direction des Etudes et Recherches (DER) d'EDF développe depuis 1991 un outil informatique d'Aide à la Gestion Intégrée de la Ressource en Eau: le logiciel AGIRE (Rietjens, 1993). La première version de ce logiciel (version 1.0) est aujourd'hui achevée : il s'agit d'une version monodimensionnelle, en mode permanent et à pas de temps mensuel.

Le produit élaboré se présente comme un outil de concertation, permettant à l'utilisateur de visualiser les conséquences, à la fois sur le milieu aquatique et sur la satisfaction des usagers, de différents scénarios d'aménagement et de gestion du bassin. Ces scénarios peuvent porter :

- sur le niveau de développement ou d'équipement des usages agricoles, industriels ou domestiques,

- sur les schémas de circulation d'eau (lâchers de barrages amont, transferts d'eau d'un milieu aquatique à un autre,...),

- sur des exigences environnementales (débits minimum admissibles en rivière ou débits de crise par exemple),

- sur les aléas climatiques.

Son objectif est de permettre de choisir entre des options d'aménagement du bassin, dans le cadre de gestions à long terme, telles que celles qui pourraient être menées dans les politiques d'aménagement des SAGE par exemple.

Cet outil informatique, qui fonctionne sur station de travail, est bâti autour :

- d'un outil de simulation reposant sur une bibliothèque de modèles numériques,

- d'un outil de visualisation développé à partir du système d'information géographique ARC INFO.

Une première application sur deux rivières du Sud-Ouest de la France (le Gave de Pau et l'Echez, deux affluents de l'Adour), réalisée dans le cadre d'une convention entre EDF-DER, l'Agence de l'Eau Adour-Garonne, le Ministère de l'Environnement et I'Institution Interdépartementale pour l'Aménagement Hydraulique du Bassin de l'Adour, est actuellement opérationnelle à l'Agence de l'Eau Adour-Garonne.

\section{L'OUTIL DE SIMULATION}

L'outil de simulation repose :

- sur une base de données environnementales 
- sur une bibliothèque de modèles numériques.

\subsection{La base de données environ- nementales}

Cette base de données spatio-temporelles contient à la fois :

- des grandeurs relatives à la description du système physique, comme des données de surface de bassins versants, la longueur ou la pente des tronçons de rivière, la morphologie (type de substrat), les données météorologiques ou les débits.

- des données liées aux activités humaines telles que:

- pour l'usage agricole, les données issues du Recensement Général Agricole (surfaces irriguées, types de culture, nombre de têtes de bétail, etc...) ainsi que des grandeurs données par différents organismes agricoles comme les ventes annuelles d'engrais par département...,

- pour l'usage domestique, les données sur la population, ainsi que les données relatives aux stations d'épuration (capacité, rendement,...)

- pour l'usage industriel, les données liées aux consommations en eau (importance, origine), aux installations de prétraitement, au rythme de fonctionnement des entreprises...

La liste indiquée n'est pas exhaustive; elle se justifie par les besoins des modèles de calcul qui sont utilisés pour estimer les demandes et rejets en eau des différents usagers et simuler les variables décrivant l'écosystème fluvial.
La constitution de cette base de données a mis en exergue le problème de la disponibilité et de la fiabilité des informations. La tâche n'est pas aisée dans la mesure où, en France, de nombreux organismes interviennent dans la gestion et le contrôle de la ressource en eau. Plusieurs bases de données existent. Lorsque qu'un paramètre est suivi par plusieurs organismes et qu'il y a incohérence, la plus grosse difficulté est de déterminer les sources les plus fiables. Mais surtout, c'est le manque de données qui pose problème et qui nécessite le lancement de programmes de mesure appropriés. Précisons que cette problématique conditionne la fiabilité de l'outil de simulation, dont les hypothèses simplificatrices ne peuvent pas toujours être choisies à bon escient, faute de données suffisantes.

\subsection{L'outil de simulation}

\subsubsection{La bibliothèque de modèles}

L'outil de simulation est constitué d'une bibliothèque de programmes numériques développés en FORTRAN qui s'enchaînent de façon modulaire. Cette bibliothèque est appelée à s'enrichir en fonction des problèmes rencontrés localement.

Le logiciel AGIRE version 1.0 (version monodimensionnelle, en mode permanent et à pas de temps mensuel) comprend aujourd'hui (fig. 1) :

- un modèle hydrologique (calcul de la répartition longitudinale du débit)

- un modèle hydraulique (calcul des vitesses et profondeur en fonction du débit) 


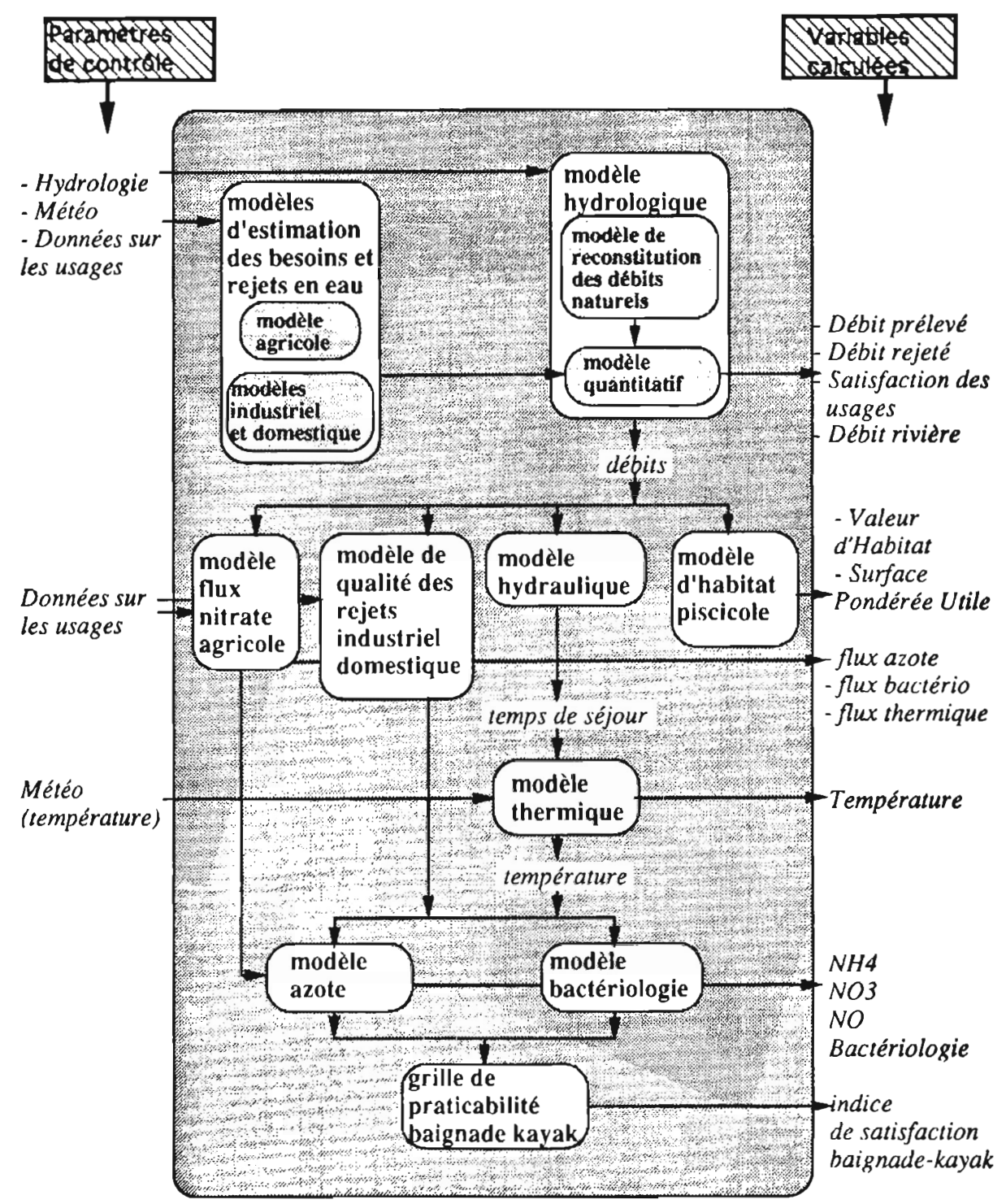

Fig. 1. - Organisation des modèles dans l'outil de simulation.

- un modèle d'estimation des besoins et rejets en eau pour l'irrigation

- un modèle d'estimation des besoins et rejets en eau pour les usages industriel et domestique (quantitatif et qualitatif) (Rietjens, Soulat, 1994)

- un modèle thermique (GOSSE, 1990) 
- un modèle de calcul des flux en nitrates liés aux pratiques agricoles sur un bassin versant (d'après Geng, 1988)

- un modèle d'azote en rivière (d'après le modèle QUAL2E, Brown et Barnwell, 1987)

- un modèle bactériologique en rivière (calcul des coliformes totaux, d'après le modèle QUAL2E, Brown et Barnwell, 1987)

- un modèle de qualité d'habitat piscicole pour la truite Fario (Sabaton et Miquel, 1993)

- un modèle d'estimation de la satisfaction pour les activités récréatives baignade et kayak.

\subsubsection{L'intervention de l'utilisateur}

Les modèles développés permettent de reproduire les logiques d'actions de l'homme sur la ressource en eau, de les modifier et ainsi d'analyser l'impact des activités humaines sur le milieu naturel.

Cette analyse se fait par secteur d'activité et par action. Un secteur d'activité génère des actions qui satisfont, ou sont la conséquence, de besoins. Ces actions se traduisent par des pressions sur la ressource en eau, tant du point de vue quantitatif que qualitatif.

Elles peuvent également conduire, par l'intermédiaire du vecteur eau, à des pressions sur d'autres secteurs d'activités, pouvant aller jusqu'à la situation de conflit d'usages.
Les actions sur la ressource en eau prises en compte dans le logiciel AGIRE sont liées :

- d'une part aux prélèvements en eau (rivière, nappe, retenue)

- d'autre part aux rejets en rivière, tant sur le plan quantitatif que qualitatif.

L'analyse des relations secteurs d'activité - actions montre que certains secteurs d'activité, tels les secteurs domestiques et industriels, sont étroitement liés; il est alors parfois difficile de bien identifier les facteurs de causalité de certains impacts, qu'ils soient quantitatifs ou qualitatifs, sur le milieu aquatique.

Le couplage réalisé dans AGIRE entre les modèles de simulation de l'activité humaine, dont la réponse est donnée sous forme de flux entrants ou sortants en rivière, et les modèles de simulation de l'écosystème fluvial, consiste alors à relier les pressions exercées par ces activités aux compartiments de l'écosystème concerné (hydrologie, qualité deau, habitat piscicole). Ceci doit permettre d'analyser :

- d'une part les impacts des pressions sur le milieu naturel, tant du point de vue spatial que temporel,

- d'autre part les actions de contrôle par secteur d'activité.

L'utilisateur va donc agir sur les flux échangés (fig. 2) en modifiant les descripteurs de :

- l'usage domestique: les prélèvements pour la production d'eau potable ont lieu en rivière ou en nappe; les rejets d'eau usées se font 
en partie en rivière, qu'ils soient épurés ou non. Dans ce cas, les variables de commande sont le nombre d'habitants, les caractéristiques de la population (permanente, saisonnière, agglomérée, éparse), le degré d'équipement en stations d'épuration, et le niveau de fonctionnement de ces stations. La qualité des rejets est calculée pour l'azote et la bactériologie.

- l'usage industriel: l'utilisateur peut jouer sur les sources d'alimentation en eau (nappe, rivière, réseau d'eau potable), sur les équipements de prétraitement, le raccordement éventuel à des stations. Le modèle prend en compte l'impact de la pollution azotée ef thermique induite par l'usage industriel.
- l'usage agricole: les paramètres de contrôle déterminant les volumes d'eau prélevés en rivière ou en nappe sont les données relatives à l'importance des surfaces irriguées, au type d'irrigation, au type de culture. Les flux polluants en nitrates d'origine agricole sont déterminés en fonction des caractéristiques de l'élevage (type d'animaux, nombre de têtes de bétail) et par les apports en engrais chimiques.

L'utilisateur a la possibilité de rajouter de nouveaux éléments tels que des usines ou des stations d'épuration, et de créer des connections entre usines, stations, villes.

II peut également imposer, en des points particuliers qu'il choisit, des dé-

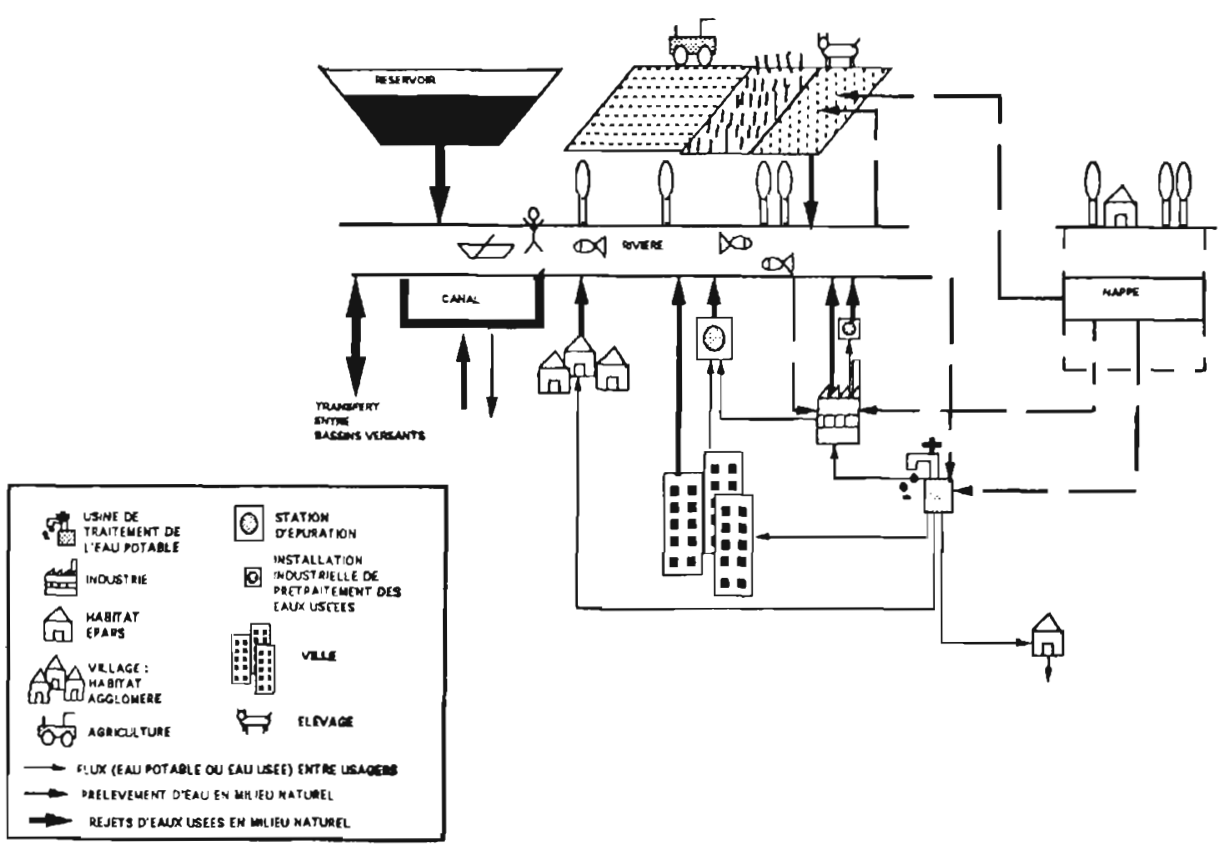

Fig. 2. - Les flux échangés entre le milieu naturel et les usagers : schéma de principe. 
bits minimums en rivière en deçà desquels tout prélèvement d'eau est interdit, ou encore simuler d'éventuels lâchers de barrages amont (par augmentation des débits aux conditions aux limites) ou des transferts d'eau entre rivières.

\subsubsection{Fonctionnement de l'outil}

Une fois les scénarios sur les usages ou sur la gestion du bassin ainsi définis, le fonctionnement global de l'ou- til de simulation peut être décrit de la façon suivante (fig. 3 ) :

A partir d'un scénario météorologique connu, un premier modèle reconstitue, sur un ou plusieurs cycles annuels, les débits dits "pseudonaturels" du cours d'eau, qui seraient les débits de la rivière ne subissant, dans le secteur de modélisation, aucun prélèvement ni rejet lié aux activités humaines. Ces calculs sont menés en chaque tronçon élémen-

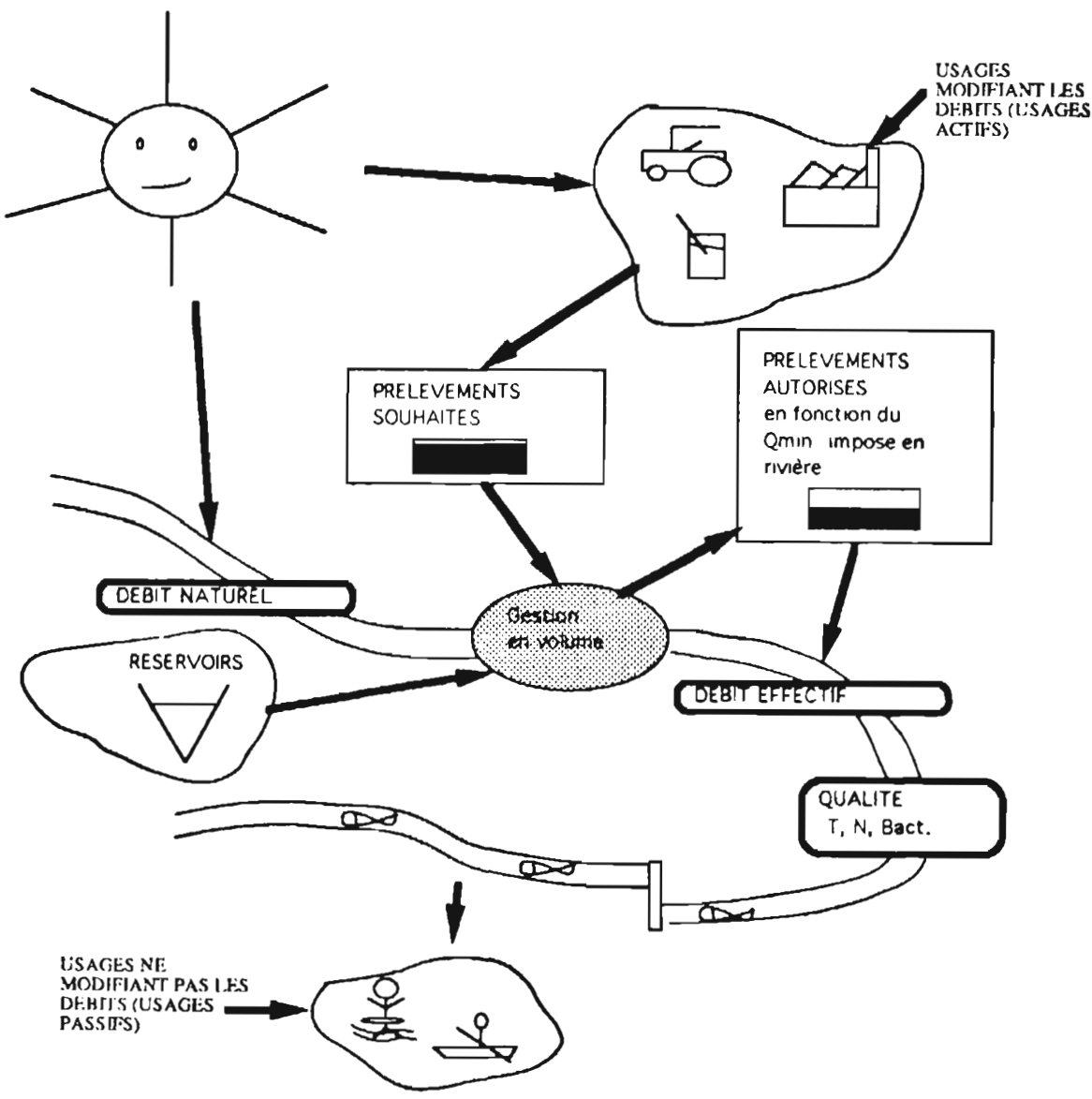

Fig. 3. - Schéma conceptuel de l'outil de simulation. 
taire (ou maille, d'une longueur moyenne égale au kilomètre) de la rivière. En parallèle, et en fonction du même scénario météorologique, on calcule, pour un état donné des usages, les besoins en eau "souhaités " pour chacun des usages dits actifs vis-à-vis de la ressource, à savoir l'usage agricole, l'usage industriel, l'usage eau potable qui influencent le débit en rivière. Sont également pris en compte, à ce niveau, les scénarios de gestion du type soutien d'étiage.

Un module de "gestion en volume" permet alors de comparer l'eau disponible dans le cours d'eau et les besoins des différents usagers. En fonction d'un ou de plusieurs débits minimums imposés dans la rivière (débit de salubrité dont on choisit la valeur en n'importe quel point), ceuxci peuvent être totalement ou partiellement satisfaits. Deux types de réponse sont obtenus à ce stade : les débits effectivement prélevés et rejetés par les différents usagers et qui, par comparaison avec la demande, donnent le degré de satisfaction de l'usage, et les débits "réels" en rivière, c'est-à-dire découlant de ce niveau d'activité humaine.

II est alors possible de calculer:

- I'hydraulique du cours d'eau (vitesse et hauteur d'eau moyennées sur des tronçons donnés)

- les variables retenues pour décrire la qualité d'eau, en tenant compte de la qualité des rejets.

- la qualité piscicole de la rivière.

En final, et sur la base des calculs précédents, il est possible de donner une note de "praticabilité" pour les activités récréatives telles que la baignade ou le kayak, en se basant sur les grilles d'autorisation utilisées par l'Administration.

Le mode de calcul est permanent pour l'ensemble des mécanismes, sauf pour l'aspect agricole où l'on tient compte dans le calcul de la variation de la réserve utile du sol d'un mois à l'autre.

Dans la version 1.0 d'AGIRE, le pas de temps est égal au mois. $\mathrm{Ce}$ choix de l'échelle de temps résulte du souci de limiter le temps de calcul afin de satisfaire la contrainte de convivialité imposée à l'outil. Cette contrainte implique également une simplification, par rapport à l'existant, de la représentation de certaines variables de calcul.

Néanmoins, la rapidité actuelle des calculs (une simulation pour une période d'une année, réalisée sur station de travail HP 7000, dure environ 2 minutes) et les progrès constants des nouveaux calculateurs devraient nous permettre à court terme de descendre à une échelle temporelle plus fine.

Enfin, une des caractéristiques d'AGIRE est de donner une description spatiale, à l'échelle d'une rivière, des conséquences d'une gestion ou d'un scénario d'aménagement du bassin. AGIRE en effet prend en compte l'impact successif des prélèvements et des rejets depuis l'amont du cours d'eau jusqu'à l'aval. Ceci permet par exemple d'étudier l'impact sur la ressource de la distribution géographique des différents usagers. Cet aspect 
n'est pas neutre en terme d'intérêt collectif, puisque l'état de l'écosystème ou la satisfaction des différents usagers dépend directement de la localisation, les uns par rapport aux autres, des différents types de cultures, des infrastructures de dépollution ou de prise d'eau, etc...

\section{L'OUTIL DE VISUALISATION}

L'outil de visualisation est construit à partir du système d'information géographique ARC INFO. II est constitué d'une base de données géographiques et d'une interface utilisateur reposant sur un modèle conceptuel de fonctionnalités.

\subsection{La base de données cartogra- phique}

La complexité du site d'étude est décomposée en couvertures (ou thèmes), chaque couverture étant porteuse d'une information - géographique (position de l'objet dans l'espace) topologique (position relative des différents objets) - descriptive (valeurs des grandeurs caractéristiques issues de la base de données précédente ou valeurs des variables de calcul attachées à cette couverture).

La version 1.0 d'AGIRE distingue les couvertures:

— villes

- réseau hydrographique local

- noms des villes

- noms des rivières

- point kilométrique

— tronçons de rivière étudiés
- agriculture

- industries

- collectivités

- stations d'épuration

- stations de traitement d'eau potable (alimentées par la rivière).

Nous avons utilisé le principe de la segmentation dynamique pour décrire la rivière, ce qui permet de lui attacher une notion de mesure (point kilométrique ou PK). Cette mesure étant définie, tout événement sur la rivière peut être décrit par son PK. Les points de prélèvement et de rejet sont alors définis comme autant d'événements ponctuels, tandis que les variables hydrauliques et de qualité, calculées maille par maille, sont identifiées comme des événements linéaires.

Les bases numériques de données géographiques nécessaires à l'outil n'étant pas disponibles sur le marché français au moment de la réalisation du prototype d'AGIRE, toutes les cartes nécessaires à la visualisation sur la zone d'application Gave de PauEchez (réseau hydrographique, secteurs hydrographiques, villes, barrages, usines, etc..) ont été digitalisées et mises en forme manuellement.

\subsection{L'interface utilisateur}

L'outil de visualisation confère au logiciel AGIRE sa convivialité. Cette interface permet à l'utilisateur de "piloter " l'outil de simulation de façon transparente en:

- construisant le scénario de simulation (choix de l'aléa météorologique, choix d'un scénario sur les 
usages, choix d'un scénario de gestion) ;

- définissant ses sorties graphiques (cartes, graphes, extension géographique, pas de temps) ;

- utilisant différentes fonctionnalités (choix des échelles, zoom,...) ;

- accédant par simple action sur la souris à des informations relatives aux usages ou à la rivière.

L'écran est partagé en quatre zones de travail (fig. 4):

- une barre de menus déroulants permettant l'accès aux différentes fonctionnalités de l'outil ;

- un écran pour la représentation cartographique de la zone d'étude ;

- un écran pour la représentation graphique temporelle;

- un écran pour la représentation graphique spatiale.

La visualisation, pour un pas de temps donné, des variables de calcul est obtenue par l'affichage simultané sur l'écran de:

- la carte de la zone d'étude avec une représentation de la variable calculée à l'aide d'une échelle de couleur:

- un graphe $(X, Y)$ représentant l'évolution de la variable sélectionnée en fonction du point kilométrique de la rivière;

- un graphe $(X, T)$ représentant l'évolution de la variable sélectionnée à l'exutoire de la rivière et sur une période annuelle. II suffit, pour tracer ce graphe en un point quelconque de la rivière, de cliquer à partir de la carte sur le point souhaité.

Pour obtenir une information sur un usage quelconque, il suffit de cliquer sur l'icône qui le symbolise. Différentes fonctionnalités permettent à l'utilisateur de changer de pas de temps, de choisir une ou plusieurs variables de calcul, de zoomer sur un graphe ou la carte, de modifier les échelles, de sauvegarder un style de représentation, de consulter les tableaux de résultats, etc...

\section{APPLICATION AU GaVE DE PAU ET À L'ECHEZ}

\subsection{Le site d'étude}

Une première application a été faite sur deux rivières du Sud-Ouest de la France, le Gave de Pau et l'Echez, situées dans le bassin de l'Adour (fig. 5).

II s'agit d'une région qui connaît épisodiquement des problèmes sévères de pénurie d'eau, liés essentiellement à une forte demande en eau d'irrigation. Le site d'étude retenu offre en fait un paysage très contrasté, tant d'un point de vue hydraulicité que d'un point de vue utilisation du bassin versant.

Sur le tronçon étudié (PierrefittePau), le Gave de Pau ne connaît pas de problèmes de pénurie en eau : son régime hydraulique, fortement influencé par les équipements hydroélectriques amont, présente un module moyen en période d'étiage au Pont- 


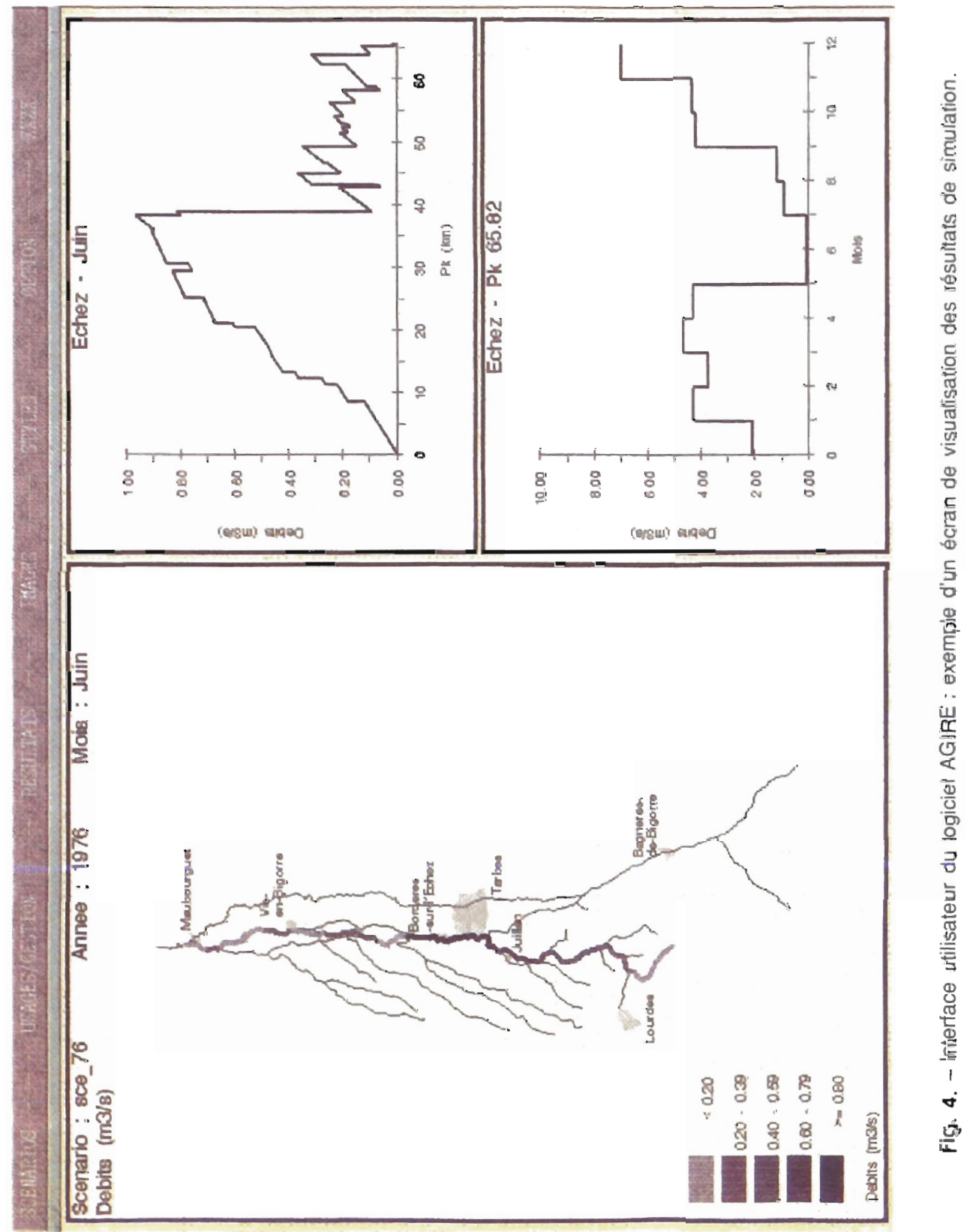




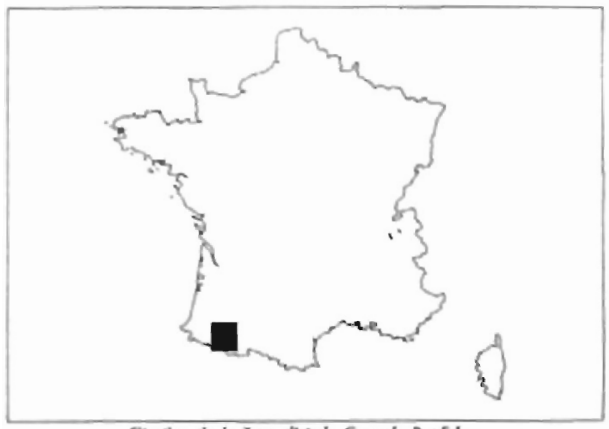

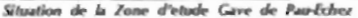

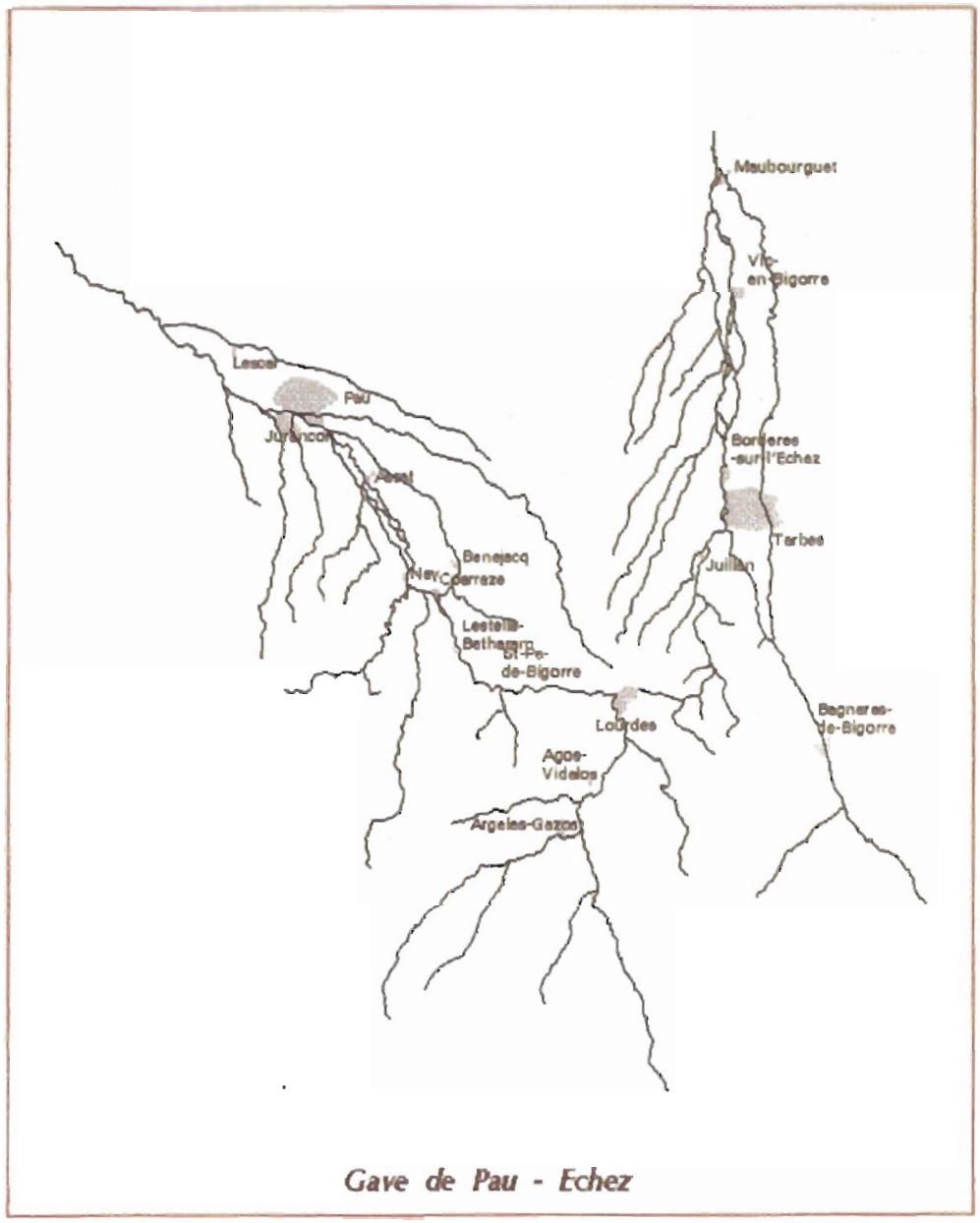

Flg. 5. - Carte de situation du site d'application Gave de Pau-Echez. 
de-Rhieulès (aval de Lourdes) de l'ordre de $20 \mathrm{~m}^{3} / \mathrm{s}$. Les besoins en eau pour les usages industriels et domestiques sont prédominants face à une demande agricole pour l'eau d'irrigation relativement faible (les surfaces irriguées ne représentent qu'à peine $3 \%$ de la totalité des surfaces cultivées sur le bassin versant étudié, (données issues du Recensement Général Agricole de 1988)). L'activité touristique y est importante, notamment autour de la région de Lourdes. La pratique du kayak est très développée sur le Gave de Pau.

Le développement de l'irrigation sur le bassin versant de l'Echez est par contre important puisque les surfaces irriguées représentent près du quart de la totalité des surfaces agricoles exploitées. L'Echez, avec un module moyen en période estivale de l'ordre de $2 \mathrm{~m}^{3} / \mathrm{s}$ à Maubourguet, à la confluence avec l'Adour, ne peut pas toujours pourvoir à la demande. Certaines années, la partie aval de l'Echez, dont l'hydraulicité est rendue très complexe du fait de la présence de nombreux canaux d'irrigation, peut s'assécher totalement.

\subsection{Exemples de scénarios}

Nous allons illustrer l'utilisation de la version 1.0 du logiciel $A G I R E$ au travers de la comparaison de quelques scénarios de simulation. Ces comparaisons vont permettre de montrer comment une hypothèse sur l'évolution à long terme d'un usage ou sur un schéma de circulation d'eau peut influencer l'état quantitatif ou qualitatif de la rivière ou le degré de satisfaction des usagers du bassin.

Une première simulation, effectuée sur une période d'un an avec les conditions météorologiques de l'année 1976 et la base de données de référence (état des usages pour l'année 1989), constitue la situation de référence. Toutes les comparaisons sont ensuite faites avec les conditions météorologiques de cette année 76 , les résultats étant présentés ici seulement pour le mois de juin.

Les différents scénarios testés sont les suivants:

- multiplication d'un facteur 5 des surfaces irriguées sur l'Echez (à hauteur de la Surface Agricole Utile);

- augmentation du rendement de toutes les stations d'épuration à hauteur de $100 \%$;

- hypothèse d'un transfert d'eau de $3 \mathrm{~m}^{3} / \mathrm{s}$ entre le Gave de Pau et l'Echez pour les mois de juin, juillet, août et septembre.

\subsection{1 scénario sur les surfaces irriguées}

La figure 6 , qui présente l'évolution des débits de l'Echez pour le mois de juin 76, met en évidence l'impact quantitatif d'une hypothèse d'évolution de la demande en eau agricole sur le régime hydraulique de la rivière. Alors que, dans la situation actuelle et pour un événement climatique particulièrement difficile comme celui de juin 1976, l'Echez connaît déjà des problèmes d'assèchement, on ne peut que constater la dégradation 

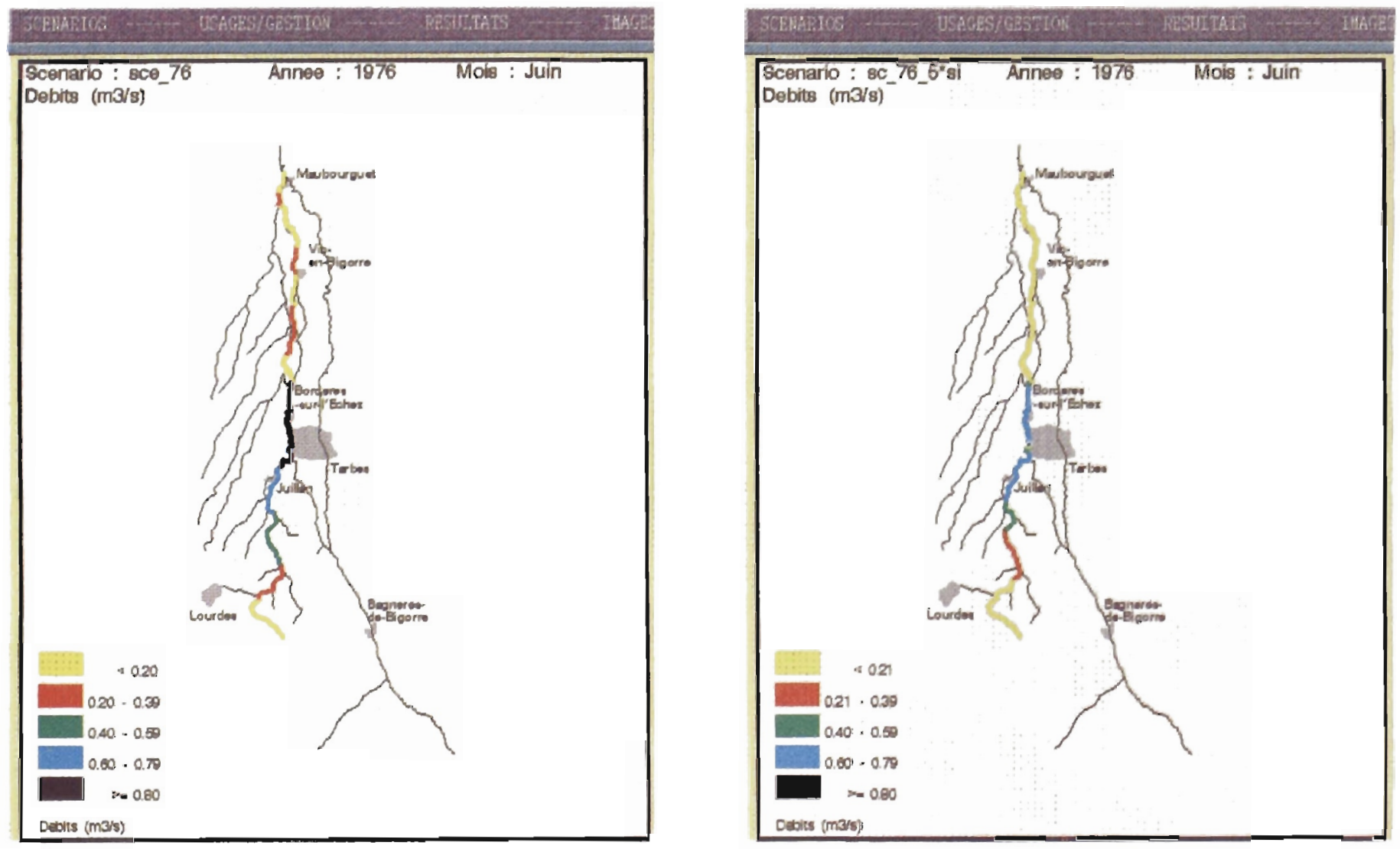

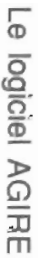

Fig. 6. - Comparaison des débits de l'Echez pour le mois de juin 1976 entre - la situation de référence

- le scénario sur les surfaces irriguées

(hypothèse d'une multiplication par 5 des surfaces îrriguées). 
de cet état avec l'hypothèse d'une croissance des surfaces irriguées d'un facteur 5. Un tiers de l'Echez, correspondant à la partie aval supportant l'ensemble des canaux d'irrigation, présente un débit inférieur à $200 \mathrm{l} / \mathrm{s}$. Il est clair qu'une telle hypothèse de demande en eau n'est pas compatible avec la capacité naturelle de cette rivière.

La figure 7 présente le degré de satisfaction de la demande en eau agricole (calculé, rappelons-le, par comparaison entre la demande en eau d'irrigation et les prélèvements effectivement réalisés, compte-tenu de la ressource disponible). Elle illustre l'impact d'une hypothèse d'évolution de la demande en eau agricole sur la pratique de cet usage. On constate que, alors que dans la situation de référence, l'ensemble des secteurs agricoles de l'Echez, à l'exception de deux d'entre eux, avaient un taux de satisfaction supérieur à $80 \%$, la nouvelle donne sur les surfaces irriguées fait chuter ce taux de façon importante pour la quasi-totalité des secteurs. AGIRE permet donc de chiffrer quantitativement cet impact.

\subsection{2 scénario sur les stations d'épuration}

Ce scénario, où l'on suppose que les stations d'épuration permettent un traitement à $100 \%$ des rejets azotés, illustre l'impact sur la qualité du milieu d'une hypothèse d'évolution d'un usage de l'eau. Cet impact est visualisé sur la figure 8 , en prenant pour exemple la concentration ammo- niacale. La comparaison entre la situation de référence et celle du scénario permet de bien mettre en évidence le rôle des rejets azotés d'origine urbaine sur le niveau de pollution en rivière. En effet, alors que dans la situation de référence, on observe sur l'Echez une dégradation des concentrations ammoniacales à partir du point de rejet de la ville de Tarbes, l'état de la rivière est fortement amélioré avec les hypothèses de ce nouveau scénario. A l'exception d'un tronçon circonscrit autour de l'agglomération de Vic-En-Bigorre, les concentrations ammoniacales descendent alors en dessous d'un seuil de $0,25 \mathrm{mgN} /$.

\subsection{3 scénario sur un transfert d'eau entre le Gave de Pau et l'Echez}

Un projet de transfert d'eau entre le Gave de Pau et l'Echez, hypothèse envisagée il y a quelques années pour les périodes d'étiage afin de combler les déficits en eau de l'Echez et surtout de l'Adour, est testé.

On suppose ici que l'on impose un transfert d'eau de $3 \mathrm{~m}^{3} / \mathrm{s}$ entre le Gave de Pau et l'Echez pour les mois de juin, juillet, août et septembre. Le point de départ, choisi arbitrairement, est situé en amont de Lourdes sur le Gave de Pau. Le point d'arrivée sur l'Echez se situe juste avant la traversée de la ville de Tarbes. Ce transfert est matérialisé sur les cartes 9 et 10 par un trait rectiligne.

Les résultats de ce scénario sont illustrés au travers de la variable tem- 

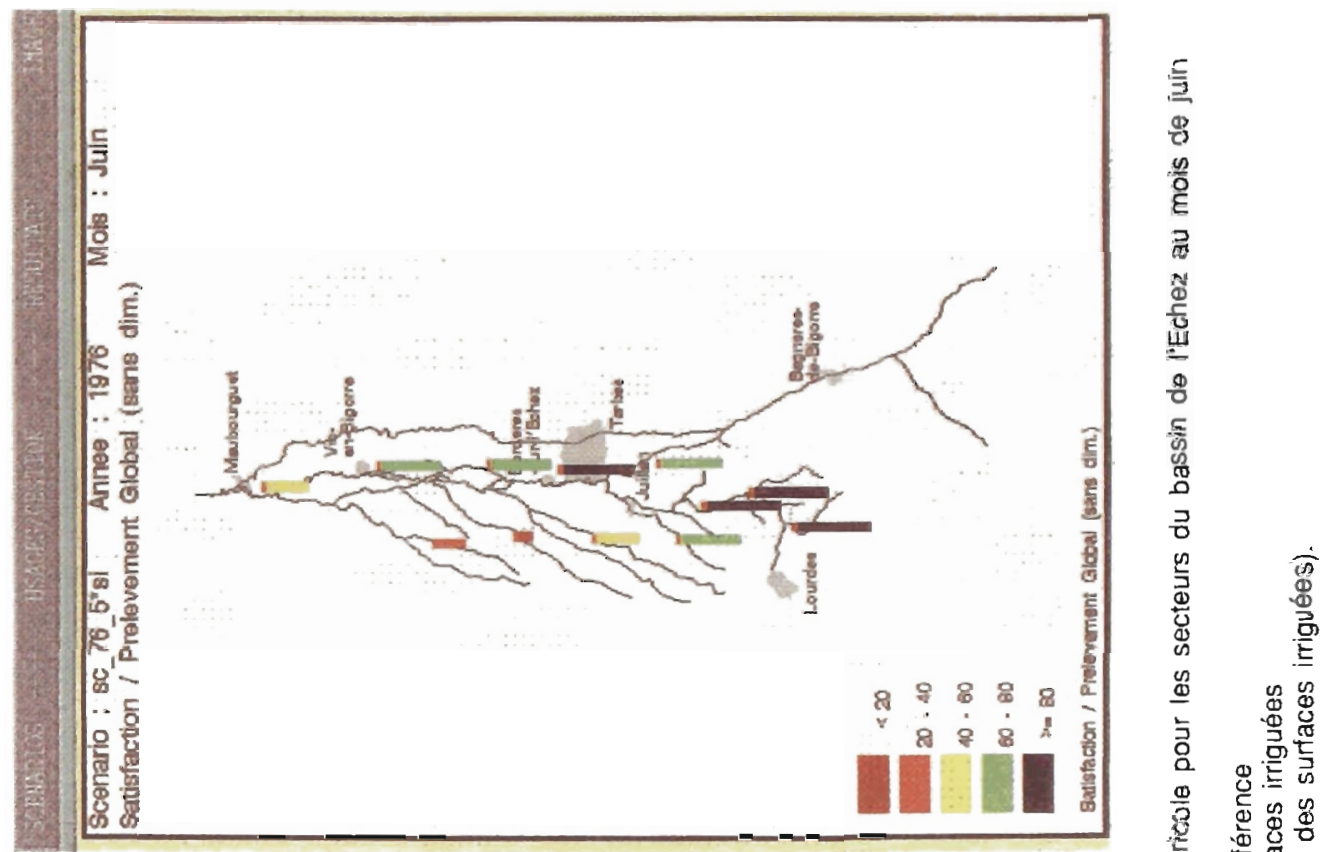

웜용

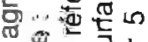

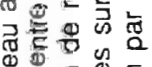
둥ㄷㅇ은은

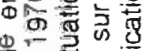

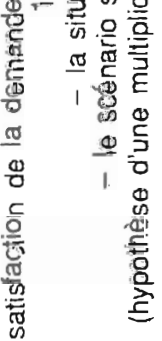

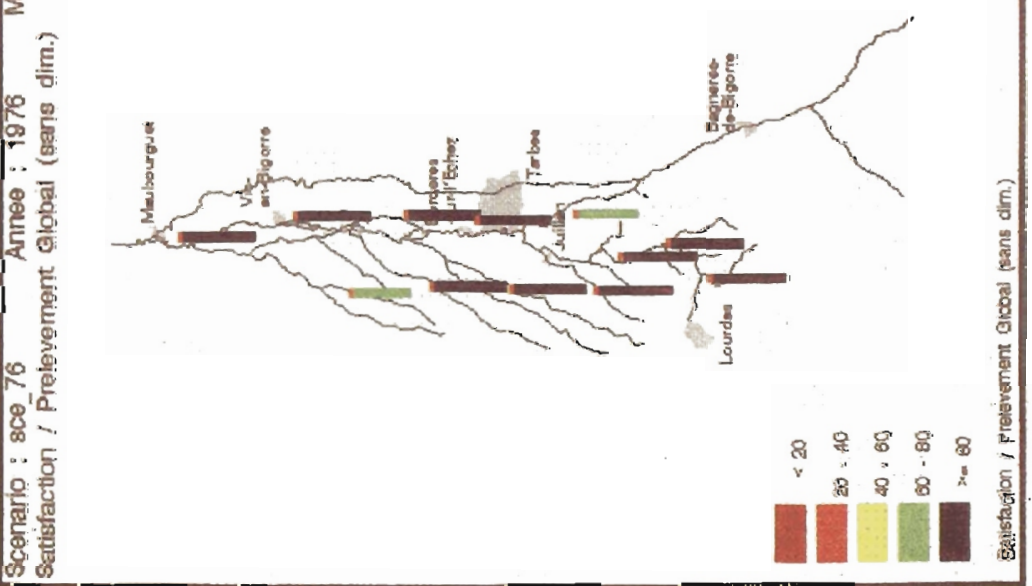

ฮ

인

क్

궁

전 1 i் 

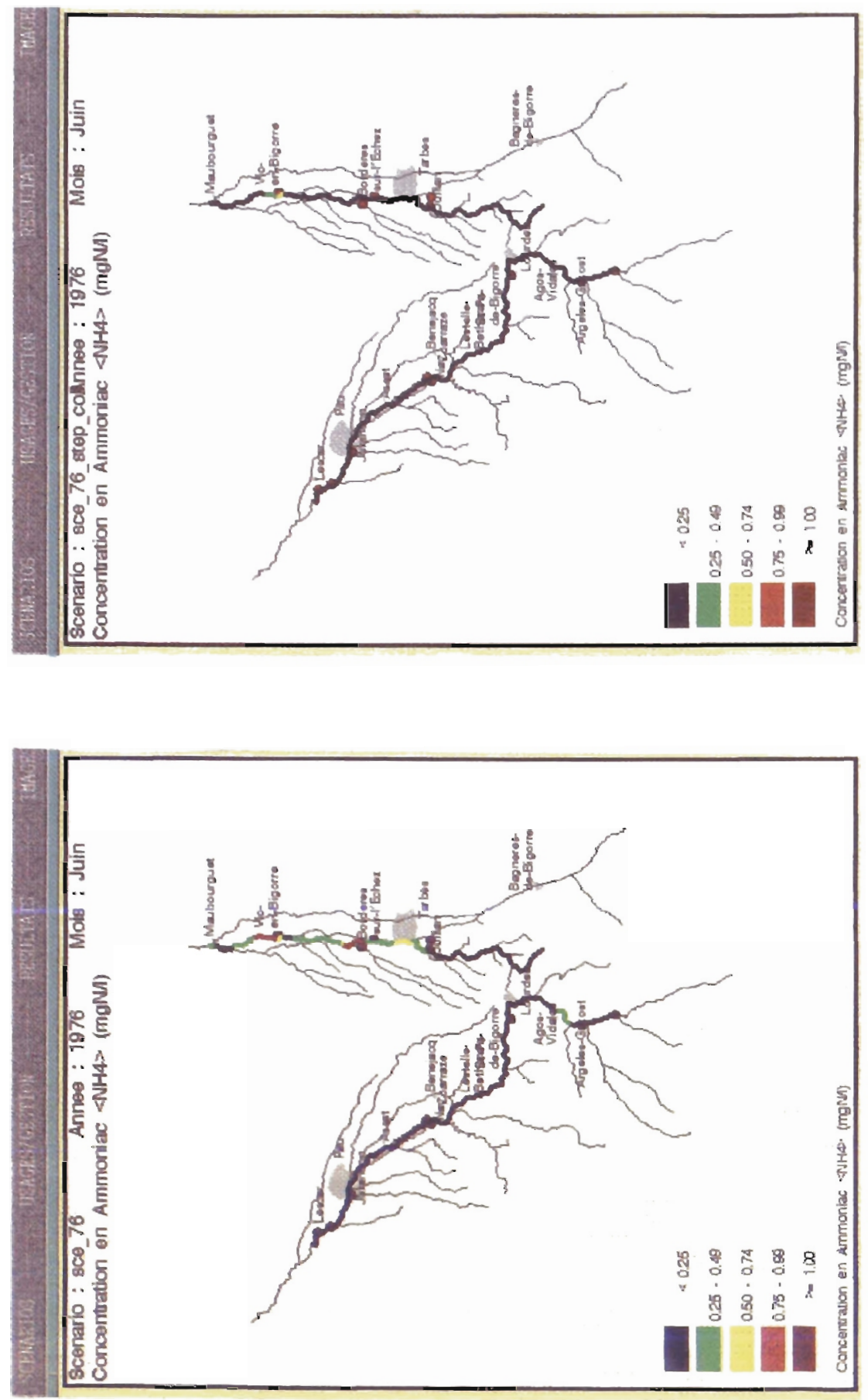

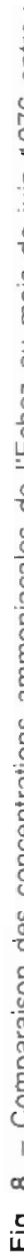


pérature en rivière (impact qualitatif d'un scénario de circulation d'eau sur le milieu aquatique) et de la variable satisfaction de la demande en eau agricole (impact d'un scénario de circulation d'eau sur la pratique d'un usage).

La figure 9 met en évidence l'impact thermique de l'arrivée des eaux plus froides du Gave de Pau dans celles de l'Echez. Au point de confluence, la chute de température est de l'ordre de $2^{\circ} \mathrm{C}$ avec les conditions météorologiques de juin 1976 et la baisse de température est encore de l'ordre de $1,5^{\circ} \mathrm{C} 40 \mathrm{~km}$ en l'aval.

Par comparaison avec la situation de référence (voir figure 10), on constate que l'hypothèse de transfert d'eau a permis de mieux satisfaire la demande en eau agricole pour les secteurs situés à l'aval du point d'arrivée supposé du canal. Bien-sûr, le secteur agricole, dont le degré de satisfaction n'était pas compris dans la tranche $80-100 \%$ en situation de référence mais qui est localisé à l'amont du transfert, n'a pas vu sa demande mieux satisfaite. ples:

On peut ainsi multiplier les exem-

- d'analyse des effets pour un scénario donné (par exemple pour les scénarios proposés, impact du transfert sur la qualité piscicole de la rivière, ou encore impact des stations d'épuration sur la praticabilité de l'activité baignade);

- de scénarios (par exemple impact de l'imposition d'un débit minimum admissible à Vic-en-Bigorre sur le degré de satisfaction de la demande agricole, ou sur la qualité de l'eau de l'Echez).

\section{CONCLUSION}

La mise au point de la version $1.0 \mathrm{du}$ logiciel AGIRE (AGIRE-1D-permanent-mensuel) a mis l'accent sur l'interfaçage des différents types de systèmes utilisés (modèles, SIG). Outre des disciplines très diverses comme l'hydrologie, la thermique, la chimie, la modélisation, cet outil a fait largement appel au génie logiciel. Le passage de l'outil "scientifique" à l'outil de "concertation " et le manque de données disponibles nous ont conduits à faire des arbitrages en simplifiant notamment la représentation de certaines variables de calcul. La lecture des résultats doit donc se faire avec toutes les réserves liées aux limites de fiabilité des modèles utilisés.

Plusieurs projets de développement sont actuellement à l'étude, notamment :

- l'enrichissement de la bibliothèque de programmes par des modèles de:

- valorisation économique pour l'irrigation ;

- dynamique de population de truites ;

- gestion des migrateurs ;

- mise au point d'une version monodimensionnelle, en mode non permanent et à pas de temps journalier ; 

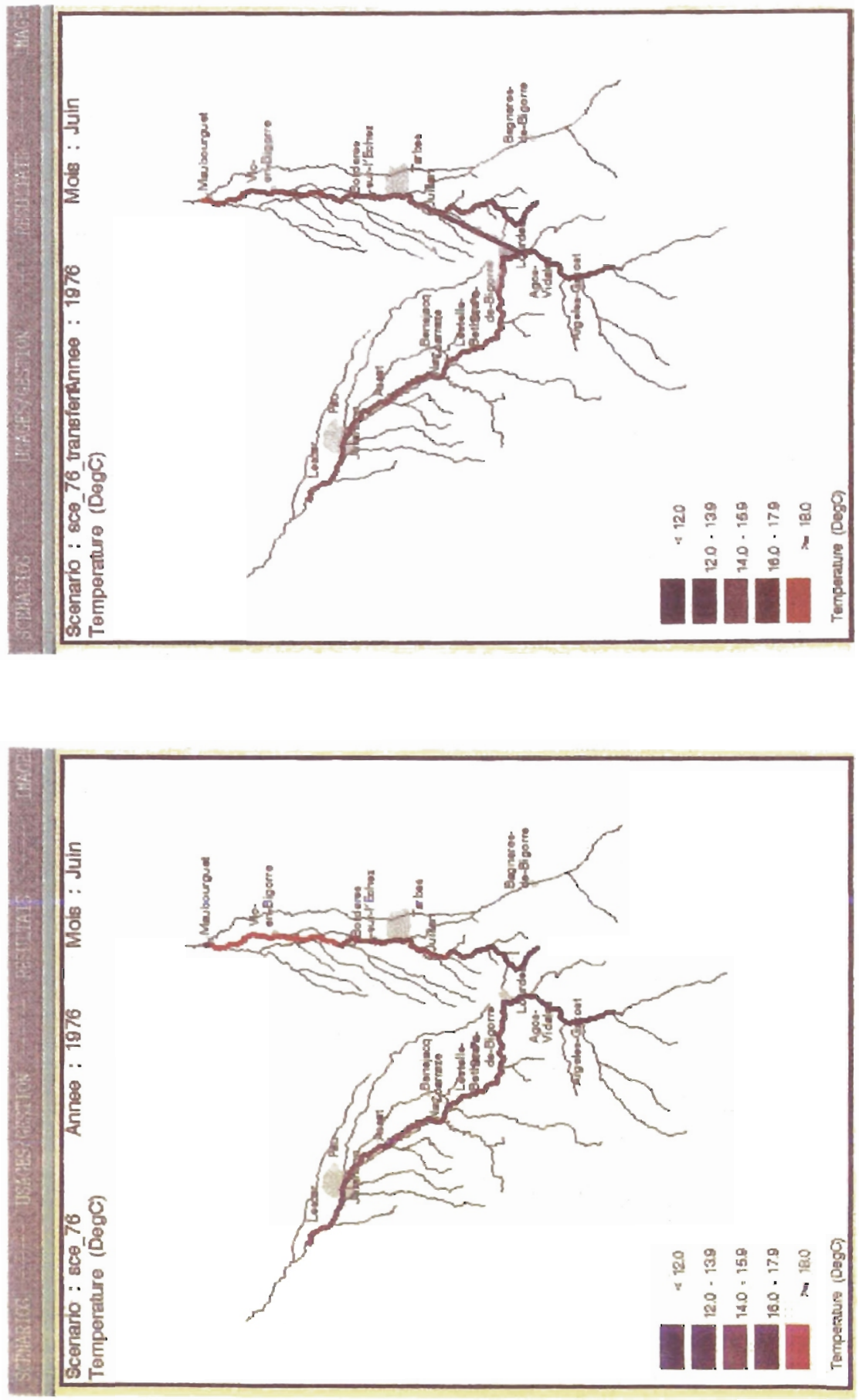

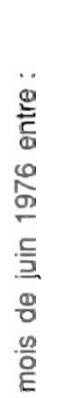



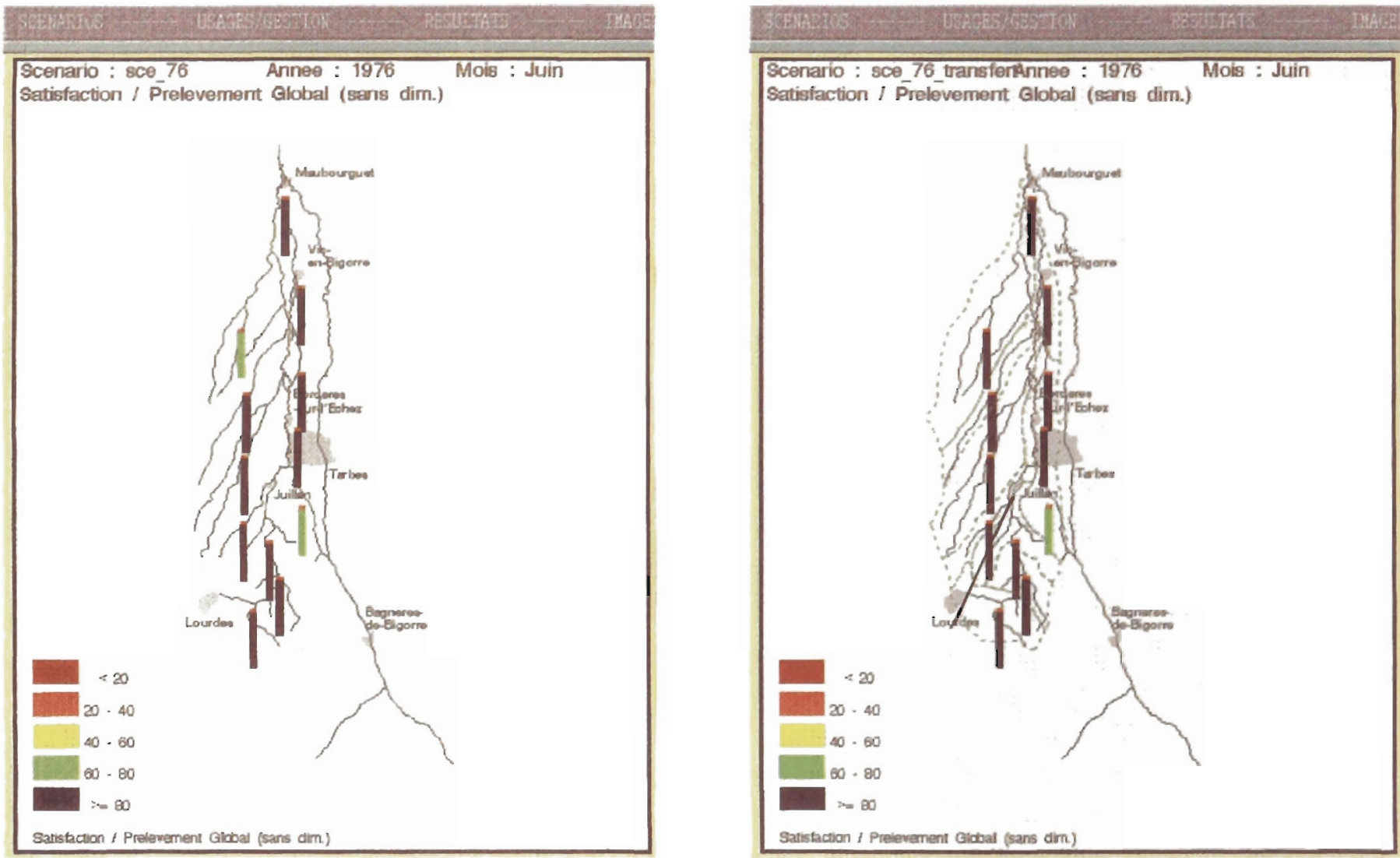

$\frac{\Gamma}{0}$
$\frac{0}{\circ}$
$\frac{\bar{\sigma}}{\Phi}$
$\frac{0}{3}$
$\frac{0}{\pi}$

Fig. 10. - Comparaison du degré de satisfaction de la demande en eau agricole pour les secteurs du bassin de rEchez au mois de juin 1976 entre :

- la situation de référence

- le scénario de transfert d'eau de $3 \mathrm{~m}^{3} / \mathrm{s}$ entre le Gave de Pau et l'Echez. 
- l'extension du domaine d'application d'AGIRE à l'échelle d'un bassin, ce qui suppose la modélisation couplée des réservoirs et des rivières.

Est également à l'étude un projet de présentation de AGIRE sous la forme d'un jeu de simulation, afin de sensibiliser le grand public aux problèmes de gestion de la ressource en eau, et de le convaincre de la nécessité de comprendre les besoins de chacun pour mener à bien le dialogue.

En fait, les voies de développement sont vastes et dépendent des questions qui peuvent être soulevées localement. Grâce à sa "modularité ", cet outil doit être un moyen performant pour aider les gestionnaires à définir, au niveau local, leurs stratégies d'aménagement à long terme et à ouvrir le dialogue entre les différents usagers de l'eau.

\section{BIBLIOGRAPHIE}

Brown L.C., Barnwell TH.O., 1987. The Enhanced Stream Water Quality Models QUAL2E AND QUAL2E-UNCAS : documentation and user manual. EPA600/3-87/007, may 1987, 189 pp.
Fourcade F., Quentin F., Rietjens J., Delcambre J., Manoha B., 1993. Combining optimized management and préservation of the environment: one example hydroelectricity and irrigation. IEA, VATTENFALL, OECD. International Conference on Hydropower, Energy and the Environement, Stockholm Sweden, 14-16 june 1993.

Geng Zhi Qinq, 1988. Modélisation conjointe du cycle de l'eau et du transfert des nitrates dans un systeme hydrologique. Thèse de 3 cycle, Ecole Nationale Supérieure des Mines de Paris, $226 \mathrm{pp}$.

Gosse Ph., 1990. Projet d'aménagement hydroélectrique de la rivière Rizzanèse (Corse) : évaluation de l'impact thermique. Rapport EDF/DER/HE31/ 90.10

Rietjens J., 1993. Note de présentation du logiciel AGIRE : Outil d'Aide à la Gestion Intégrée de la Ressource en Eau. Rapport EDF/DER/HE31/93.22.

Rietjens J., Soulat 1., 1994. Logiciel AGIRE - Version 1.0: Modélisation des besoins et rejets en eau pour les usages domestique et industriel: note de principe. Rapport EDF/DER/HE31/94.19.

Sabaton C., Miquel J., 1993 : La méthode des micro-habitats : un outil d'aide au choix d'un débit réservé à l'aval des ouvrages hydroéelectriques. Expérience d'Electricité de France. Hydroéco. Appl. (1993) Tome 5, Vol. 1, pp. 127-163. 